\title{
1 A Genomic Perspective on the Evolutionary Diversification of Turtles
}

2

3 Simone M. Gable ${ }^{1 *}$, Michael I. Byars ${ }^{1 *}$, Robert Literman ${ }^{2}$, Marc Tollis ${ }^{1}$

4

$5 \quad{ }^{1}$ School of Informatics, Computing, and Cyber Systems, Northern Arizona University, PO Box

$6 \quad$ 5693, Flagstaff, AZ 86011

$7 \quad{ }^{2}$ Department of Biological Sciences, University of Rhode Island, 120 Flagg Road, Kingstown, RI,

$8 \quad 02881$

9

$10 *$ These authors contributed equally.

11

12 Corresponding author: Marc Tollis, School of Informatics, Computing, and Cyber Systems,

13 Northern Arizona University, PO Box 5693, Flagstaff, AZ, 86011; marc.tollis@nau.edu

14

15

16 Running header: Phylogenomic Heterogeneity in Turtles 


\section{GABLE AND BYARS ET AL.}

ABSTRACT

19 To examine phylogenetic heterogeneity in turtle evolution, we collected thousands of high-

20 confidence single-copy orthologs from 19 genome assemblies representative of extant turtle

21 diversity and estimated a phylogeny with multispecies coalescent and concatenated partitioned

22 methods. We also collected next-generation sequences from 26 turtle species and assembled

23 millions of biallelic markers to reconstruct phylogenies from annotated regions (coding regions,

24 introns, untranslated regions, intergenic, and others) of the western painted turtle (Chrysemys

25 picta bellii) genome. We then measured gene tree-species tree discordance, as well as gene and

26 site heterogeneity at each node in the inferred trees, and tested for incomplete lineage sorting and

27 temporal patterns in phylogenomic heterogeneity across turtle evolution. We found 100\%

28 support for all bifurcations in the inferred turtle species phylogenies. However, a number of

29 genes, sites, and genomic features supported alternate relationships between turtle taxa, and

30 some nodes in the turtle phylogeny were well-explained by incomplete lineage sorting. There

31 was no clear pattern between site concordance, node age, and DNA substitution rate across most

32 annotated genomic regions, suggesting a relatively uniform proportion of informative sites drive

33 phylogenetic inference across the evolution of turtles. We found more gene concordance at older

34 nodes in the turtle phylogeny, and suggest that, in addition to incomplete lineage sorting, an

35 overall lack of gene informativeness stemming from a slow rate of evolution can confound

36 inferred patterns in turtle phylogenomics, particularly at more recent divergences. Our study

37 demonstrates that heterogeneity is to be expected even in well resolved clades such as turtles,

38 and that future phylogenomic studies should aim to sample as much of the genome as possible.

40 Keywords: turtles, genomes, phylogeny, discordance 


\section{PHYLOGENOMIC HETEROGENEITY IN TURTLES}

\section{Introduction}

42 Next-generation sequencing has revolutionized the understanding of evolutionary relationships

43 by greatly increasing the numbers of sampled loci (Lemmon et al. 2012; Faircloth et al. 2013;

44 Edwards et al. 2016), shedding new light on the branching order of diversification across the

45 history of life. However, phylogenetic studies based on genome-scale data often contain a great

46 deal of heterogeneity in the inferred patterns (Hime et al. 2021; Lopes et al. 2021; Morales-

47 Briones et al. 2021; Singhal et al. 2021), particularly in the form of discordance among sites and

48 gene trees (Kumar et al. 2012). Gene tree-species tree discordance occurs when gene trees are in

49 conflict with the underlying species relationships, while site discordance occurs when a subset of

50 sites support different bifurcations in a species tree, and both factors can lead to biased

51 phylogenetic estimates. Biological factors driving this heterogeneity include: differing

52 evolutionary rates across the genome, incomplete lineage sorting, gene duplication or loss, and

53 hidden paralogy (Pamilo and Nei 1988; Galtier and Daubin 2008). Artifactual factors driving

54 heterogeneity include sequencing errors, as well as substitution model violations such as

55 saturation or multiple hits (Foster 2004; Cooper 2014; Cox et al. 2014). Accounting for gene and

56 site discordance often requires the application of multiple tools in phylogenetic analysis,

57 including partitioning schemes which try to account for differing evolutionary rates and model

58 parameters (Kubatko and Degnan 2007), and multispecies coalescent methods accounting for

59 population-level processes such as incomplete lineage sorting (Edwards et al. 2016). The

60 application of hundreds or even thousands of loci is often an attempt to overcome errors

61 stemming from or driving phylogenomic conflicts, with the goal of reconstructing better-

62 resolved phylogenies by reducing the overall sampling variance (Minh et al. 2020a). 


\section{GABLE AND BYARS ET AL.}

An emerging advantage of the genomic era is the theoretical ability to access the whole genome in order to sample loci representing a diversity of evolutionary rates and coalescent

65 histories, potentially overcoming problems from discordance in phylogenomics (Wolf et al.

66 2002; Rokas et al. 2003). The most widely used sampling methods include the use of large

67 numbers of coding sequences (Singhal et al. 2021), anchored hybrid enrichment loci (Lemmon et

68 al. 2012), ultra-conserved elements (Faircloth et al. 2013), and transcriptomes (Irisarri et al.

69 2017), which encompass hundreds or thousands of markers yet are still reduced representations

70 of a small proportion of the genome (Lynch 2007). The development of bioinformatics tools and

71 sequencing technologies have enabled chromosome-scale genome assemblies for non-model

72 organisms (see http://dnazoo.org, Dudchenko et al. 2017), facilitating the understanding of not

73 only which diversification events are associated with discordance, but also the genomic regions

74 or types of loci driving heterogeneous phylogenetic results.

75 Only recently have investigators looked closely at patterns of heterogeneity in order to

76 understand how molecular evolution across the genome affects inferred species relationships,

77 mainly in clades where there has been considerable phylogenetic and taxonomic debate such as

78 squamate reptiles (Burbrink et al. 2020; Singhal et al. 2021), birds (Jarvis et al. 2014; Prum et al.

79 2015), amphibians (Hime et al. 2021), and land plants (Morales-Briones et al. 2021). Meanwhile,

80 discordance in well-resolved phylogenies with high statistical support among inferred branches

81 and agreement across studies has been largely overlooked, leaving a gap in knowledge about the

82 expected range of signals and amount of disagreement between loci when there are differing

83 levels of phylogenetic uncertainty. This would be critical for informing the designs of future

84 phylogenomic studies. 


\section{PHYLOGENOMIC HETEROGENEITY IN TURTLES}

Here, we examined the importance of heterogeneity in the phylogenomic estimation of turtles (Order Testudines). Turtles are shelled reptiles with a rich fossil history dating from the

87 Triassic Period (Joyce 2007; Joyce et al. 2021). The approximately 350 extant species of turtles are classified into two main groups, Pleurodira and Cryptodira, which are further divided into 8 superfamilies and 14 families (Uetz et al. 2021). Early molecular phylogenetic results disagreed in the placement of some turtle taxa; in particular, the position of the monotypic big-headed turtle (Platysternon) and its relationships with or within Testudinoidea (Shaffer et al. 1997;

92 Krenz et al. 2005). Other questions about deeper turtle relationships such as the position of soft-

93 shell turtles (Trionychoidea), the Americhelydiae, and the position of turtles in the amniote

94 phylogeny persisted into the next-generation sequencing era (Crawford et al. 2012, 2015). Since

95 then, phylogenomic studies of turtles have produced fully resolved species trees with $100 \%$

96 statistical support at every node and near complete agreement across studies (Crawford et al.

97 2015; Shaffer et al. 2017).

Despite the harmony among phylogenomic studies of turtles using next-generation

99 sequencing data, the amount of underlying heterogeneity in these datasets has not been

100 examined. Also, while complete genome assemblies are available for a wide range of turtle

101 species (Shaffer et al. 2013; Wang et al. 2013; Tollis et al. 2017; Quesada et al. 2019),

102 phylogenomic studies have not taken advantage of this genome-wide information. Our goals

103 were threefold: (1) to revisit discoveries about higher turtle systematics using more substantial

104 proportions of the genome than previous studies; (2) to measure heterogeneity at genes and sites

105 in important events in turtle evolution; and (3) determine what drives heterogeneity in turtle

106 phylogenomics in terms of mechanisms and locus type. We show that heterogeneity in

107 phylogenomic datasets is to be expected, even in well-resolved clades such as turtles, and 


\section{GABLE AND BYARS ET AL.}

108 suggest that a combination of incomplete lineage sorting and lack of gene informativeness due to

109 an overall slow rate of evolution may be driving incongruence between studies of turtle

110 relationships.

\section{Methods and Materials}

113 Phylogenomic analysis of high-confidence single-copy orthologs

114 We downloaded the complete genome assemblies of 19 turtle species plus three outgroup taxa

115 from NCBI. The species, assembly accession numbers, assembly lengths, and assembly

116 contiguities measured by scaffold N50 are shown in Table 1. Using Benchmarking Universal

117 Single-Copy Orthologs (BUSCO) v3 (Waterhouse et al. 2018), we extracted the nucleotide

118 sequences of 5,310 OrthoDB v9 tetrapod orthologs (Zdobnov et al. 2017) from each genome

119 assembly. BUSCO orthologs are high-confidence genes that persist in eukaryotic genomes as

120 single-copy, which precludes downstream problems in phylogenetics stemming from gene

121 duplication and hidden paralogy (Waterhouse et al. 2018). We aligned each set of orthologs with

122 MAFFT v7.475 (Katoh and Standley 2013), and removed erroneous columns in the alignments

123 with the heuristic method (automated 1) in TrimAL v1.4.1 (Capella-Gutierrez et al. 2009).

124 Summary statistics including number of taxa, alignment length, missing data percentage, number

125 and proportion of variable sites, number and proportion of parsimony-informative sites, and GC

126 content for each alignment were estimated with AMAS v3.04 (Borowiec 2016). Trimmed

127 alignments were filtered at a cut-off length $\geq 1,500 \mathrm{bp}$ and a minimum of $50 \%$ taxa

128 representation. We estimated the average pairwise Kimura 2-parameter corrected distance for

129 each alignment with MEGAX (Kumar et al. 2018). 
bioRxiv preprint doi: https://doi.org/10.1101/2021.10.14.464421; this version posted October 16, 2021. The copyright holder for this preprint (which was not certified by peer review) is the author/funder, who has granted bioRxiv a license to display the preprint in perpetuity. It is made available under aCC-BY-NC-ND 4.0 International license.

\section{PHYLOGENOMIC HETEROGENEITY IN TURTLES}

Table 1: Turtle genome assemblies analyzed in this study.

\begin{tabular}{|c|c|c|c|c|c|c|}
\hline Suborder & Superfamily & Family & Species Name & Accession Number & $\begin{array}{c}\text { Assembly length } \\
\text { (Gb) }\end{array}$ & $\begin{array}{c}\text { Scaffold N50 } \\
\text { (Mb) }\end{array}$ \\
\hline \multirow[t]{15}{*}{ Cryptodira } & \multirow[t]{2}{*}{ Chelonioidea } & Cheloniidae & Chelonia mydas & GCF_000344595.1 & 2.2 & 3.9 \\
\hline & & Dermochelyidae & $\begin{array}{c}\text { Dermochelys } \\
\text { coriacea }\end{array}$ & GCA_006547105.1 & 2.1 & 0.12 \\
\hline & Chelydroidea & Chelydridae & Chelydra serpentina & GCA_007922165.1 & 2.5 & 16 \\
\hline & Kinosternoidea & Dermatemydidae & Dermatemys mawii & GCA_007922305.1 & 1.9 & 34.4 \\
\hline & \multirow[t]{8}{*}{ Testudinoidea } & \multirow[t]{3}{*}{ Emydidae } & Chrysemys picta & GCA_000241765.3 & 2.4 & 0.44 \\
\hline & & & Malaclemys terrapin & GCA_001728815.2 & 2.4 & 0.44 \\
\hline & & & Terrapene mexicana & GCF_002925995.2 & 2.6 & 24.2 \\
\hline & & \multirow[t]{2}{*}{ Geoemydidae } & Cuora amboinensis & GCA_004028625.2 & 2.1 & 0.47 \\
\hline & & & Cuora mccordi & GCA_003846335.1 & 2.4 & 32.6 \\
\hline & & Platysternidae & $\begin{array}{l}\text { Platysternon } \\
\text { megacephalum }\end{array}$ & GCA_003942145.1 & 2.3 & 7.2 \\
\hline & & \multirow[t]{2}{*}{ Testudinidae } & $\begin{array}{l}\text { Chelonoidis } \\
\text { abingdonii }\end{array}$ & GCA_003597395.1 & 2.3 & 1.3 \\
\hline & & & Gopherus agassizii & GCA_002896415.1 & 2.2 & 0.228 \\
\hline & \multirow[t]{3}{*}{ Trionychoidea } & Carettochelyidae & $\begin{array}{l}\text { Carettochelys } \\
\text { insculpta }\end{array}$ & GCA_007922185.1 & 2.3 & 45.9 \\
\hline & & \multirow[t]{2}{*}{ Trionychidae } & Apalone spinifera & GCA_000385615.1 & 1.9 & 2.3 \\
\hline & & & Pelodiscus sinensis & GCF_000230535.1 & 2.2 & 3.4 \\
\hline \multirow[t]{4}{*}{ Pleurodira } & \multirow[t]{2}{*}{ Chelidoidea } & \multirow[t]{2}{*}{ Chelidae } & Emydura subglobosa & GCA_007922225.1 & 2 & 44.8 \\
\hline & & & $\begin{array}{c}\text { Mesoclemmys } \\
\text { tuberculata }\end{array}$ & GCA_007922155.1 & 2 & 46.4 \\
\hline & \multirow[t]{2}{*}{ Pelomedusoidea } & Pelomedusidae & Pelusios castaneus & GCA_007922175.1 & 2 & 14.1 \\
\hline & & Podocnemididae & Podocnemis expansa & GCA_007922195.1 & 2.4 & 37.1 \\
\hline
\end{tabular}

$\mathrm{Gb}=$ gigabases; $\mathrm{Mb}=$ megabases 


\section{GABLE AND BYARS ET AL.}

138 al. 2018), assessing branch support with local posterior probabilities (Sayyari and Mirarab 2016).

139 We used Snakemake (Mölder et al. 2021) to automate a reproducible bioinformatic pipeline for

140 BUSCO extraction, filtering, alignment, gene tree and species tree estimation, available at

141 https://github.com/mibyars/busco_phylo_pipeline. We also concatenated the single copy

142 orthologs into a supermatrix with AMAS and performed a partitioned maximum likelihood

143 phylogenetic reconstruction with IQ-TREE v2.1.2 (Minh et al. 2020b). We used ModelFinder

144 (Kalyaanamoorthy et al. 2017) set to TESTMERGE to combine partitions where necessary. We

145 assessed branch support on the concatenated partitioned maximum likelihood tree using 1,000

146 ultrafast bootstrap replicates and the Shimodaira-Hasegawa-like (SH-like) approximate

147 likelihood ratio test (aLRT, Shimodaira 2002).

Phylogenomic analysis of biallelic markers

150 In addition to single-copy orthologs from turtle and outgroup genome assemblies, we constructed

151 sets of biallelic single nucleotide polymorphisms (SNPs) across annotated features of the turtle

152 genome based on paired-end sequence data downloaded from the NCBI Short Read Archive

153 (SRA, Supplementary Data). We assessed each short read dataset with FastQC v0.115 (S.

154 Andrews - http://www.bioinformatics.babraham.ac.uk/projects/fastqc/) and trimmed the raw

155 reads with BBDuk v.37.41 (B. Bushnell - sourceforge.net/projects/bbmap/, last accessed

156 December 2020). We used SISRS to generate de novo orthologs from the processed reads

157 (Schwartz et al. 2015; Literman and Schwartz 2021). After subsampling all sequences to a target

158 depth of $\sim 10 \mathrm{X}$ per taxon (except for two low-coverage species, Supplementary Data), we

159 assembled a composite genome using Ray v2.2.3-devel (Boisvert et al. 2010) with default

160 parameters and a k-value of 31 . We then used SISRS to map the trimmed reads from each 


\section{PHYLOGENOMIC HETEROGENEITY IN TURTLES}

161 species to the composite genome, retaining only uniquely mapped reads to avoid false positives

162 from duplicated or repetitive regions. Bases in the composite genome were replaced according to

163 mapped data from each species if sites were covered by at least three reads and if there was no

164 within-taxon variation; sites not meeting these criteria were masked as "N".

165 For downstream ortholog annotation, we mapped the composite sequences from each

166 species to the reference genome for the western painted turtle (Chrysemys picta bellii,

167 GCA_000241765.3) with Bowtie2 v2.3.4 (Langmead and Salzberg 2012), removing contigs that

168 either failed to map or mapped to multiple positions, and removing sites resulting from

169 overlapping coverage of independent scaffolds. We then annotated the mapped composite

170 genome sites parsed from the painted turtle genome as belonging to the following genomic

171 regions (locus types) with BEDTools v2.2.6 (Quinlan and Hall 2010): (1) coding sequences

172 (CDS); (2) 3'-UTR; (3) 5'-UTR; (4) introns; (5) other genic regions not annotated as CDS, UTR,

173 or intronic; (6) long-noncoding RNAs (lncRNAs); (7) pseudogenes; (8) smRNAs (including

174 miRNAs, ncRNAs, rRNAs, scRNAs, smRNAs, snoRNAs, snRNAs, tRNAs, and vaultRNAs).;

175 and (9) intergenic regions. We defined "intergenic" as all regions not included within the other

176 locus types.

177 We then used SISRS to identify parsimony-informative sites along the mapped composite

178 contigs and to remove invariant sites and singletons, as well as any sites containing "Ns" (i.e.

179 polymorphic within species) and overlapping indels or gaps. For the nine annotated genomic

180 regions as well as a set of the complete mapped data (=10 locus types), we concatenated biallelic

181 parsimony-informative sites and inferred locus type phylogenies via SVDQuartets (Chifman and

182 Kubatko 2014) in PAUP* v4.0a (build 168) (Swofford 2003) using the default 100 bootstrap

183 replicates to estimate a majority consensus tree, and maximum likelihood inference in IQ-TREE. 


\section{GABLE AND BYARS ET AL.}

184 A schematic of all bioinformatic analyses used to extract homologous markers is found in Figure

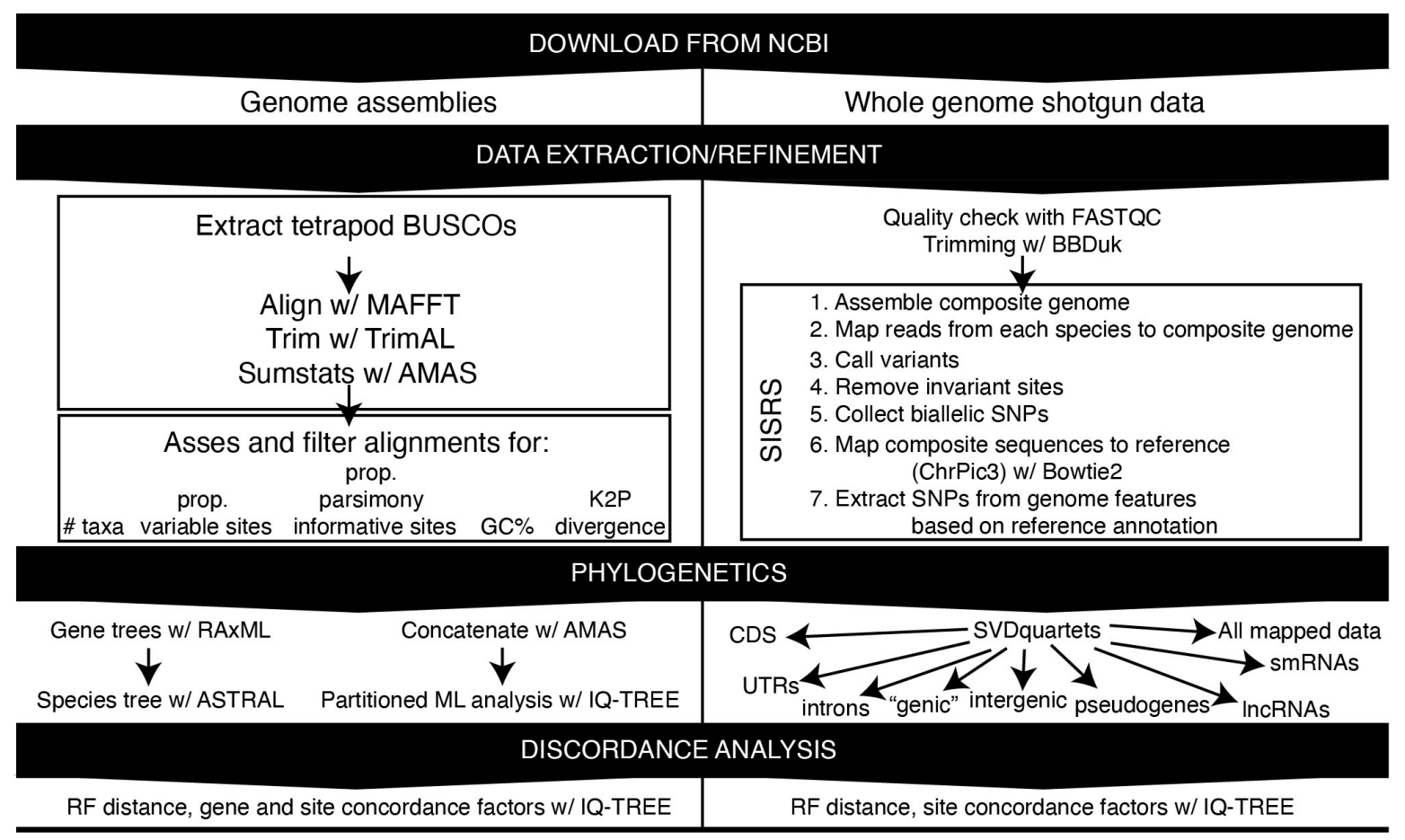

Figure 1. Bioinformatics pipelines. Schematic representing the workflows used in this study to extract homologous

189 markers from genome assemblies and whole genome shotgun sequences for phylogenomic inference of turtles.

$190 \mathrm{BUSCO}=$ Benchmarking Single Copy Orthologs; $\mathrm{ML}=$ maximum likelihood; $\mathrm{RF}=$ Robinson-Fould's.

\section{Heterogeneity analyses}

193 To measure similarity across gene genealogies, we calculated Robinson-Foulds (RF) distances

194 (Robinson and Foulds 1981) with PhyKIT (Steenwyk et al. 2021). First, we measured the RF

195 distance between each inferred single-copy ortholog gene tree and the inferred species tree. In

196 order to take into account missing species across some of the gene trees, we normalized the RF

197 distances by taking the raw RF distance and dividing it by $2(n-3)$ where $n$ is the number of tips in

198 the phylogeny. We also calculated multiple pairwise RF distances between the locus type 


\section{PHYLOGENOMIC HETEROGENEITY IN TURTLES}

phylogenies inferred from data mapped to each genome annotation feature with SISRS (CDS, introns, intergenic, etc.). Finally, we calculated the RF distances of each locus type phylogeny from the topology inferred with all mapped sites across the turtle genome.

We computed gene and site concordance factors for each branch in the turtle phylogeny using the trimmed and filtered single-copy ortholog alignments, their gene trees, and the coalescent-based species tree in IQ-TREE (Minh et al. 2020a). A branch's gene concordance factor represents the percentage of decisive gene trees that also contain that branch, and a branch's site concordance factor is the percentage of decisive alignment sites supporting that branch. We also computed gene concordance factor and site concordance factor for each branch in the concatenated single-copy ortholog tree of alignments $>1,500$ bp by computing individual locus trees based on the partitions in IQ-TREE. Locus trees were inferred with default settings, and the species tree was inferred using an edge-linked proportional partition model with an SHlike approximate likelihood ratio test and ultrafast bootstrap analysis of 10,000 replicates each (alrt 10000 -B 10000). The greedy algorithm of PartitionFinder (Lanfear et al. 2012) was applied to find the best-fit partitioning scheme which was then used in the subsequent tree reconstruction step (-m TESTMERGE). We also calculated site concordance factor for every branch in the maximum likelihood trees inferred from biallelic parsimony informative sites within locus types. Concordance factors for genes and sites allow for complementary approaches to measure the consistency of loci that agree with particular branches in a phylogeny, as well as opportunities to test for incomplete lineage sorting, which we applied to each branch in the single-copy ortholog species tree. One expectation of incomplete lineage sorting is a roughly equal proportion of topologies supporting alternative relationships (Green et al. 2010). We used a chi-square test to determine if similar proportions of gene trees supported conflicting 


\section{GABLE AND BYARS ET AL.}

222 relationships at each branch, where a failure to reject the null hypothesis supported incomplete

223 lineage sorting at a given branch.

Divergence Time Estimation

226 We estimated divergence times with single-copy ortholog alignments using Bayesian modeling

227 implemented in BEAST v.2.6.3 (Bouckaert et al. 2019). We subsampled 685 alignments with

228 complete taxon sampling, from which we further randomly sampled three replicate sets of 10

229 genes. Based on model testing with ModelFinder, we applied the HKY+Gamma+Invariant site

230 model to all partitions, and utilized a relaxed lognormal molecular clock and Calibrated Yule tree

231 prior. To calibrate the time trees, we used 11 calibration priors from the literature (Joyce et al.

232 2013) which overlapped with our taxon sampling (Supplementary Data). To account for

233 uncertainty in fossil dating, we used hard minimum constraints and set soft maximum constraints

234 by placing the maximum ages in the $97.5 \%$ quantile of a lognormal prior distribution. Markov

235 chain Monte Carlo (MCMC) analyses were sampled every 50,000 generations. For each

236 replicate, we assessed convergence of parameter estimates across the MCMC by monitoring

237 effective sample sizes with Tracer v.1.7.2 (Rambaut et al. 2018), and we estimated a maximum

238 clade credibility tree with TreeAnnotator v2.6.3.

239 We also estimated divergence times in turtle evolution using penalized likelihood with

240 r8s v1.8.1 (Sanderson 1997, 2002, 2003), using the same fossil calibrations as above for

241 minimum and maximum node ages; the main difference in this analysis was we fixed the time to

242 most recent common ancestor (TMRCA) for amniotes at 312 million years ago (Ma) (Donoghue

243 and Benton 2007) and the TMRCA for Testudines at $220 \mathrm{Ma}$ (Thomson et al. 2021). First, we

244 concatenated the 685 complete partitions and inferred a maximum likelihood tree with model 


\section{PHYLOGENOMIC HETEROGENEITY IN TURTLES}

245 testing for each partition as above in IQ-TREE. We then used the inferred phylogeny with

246 branches in terms of substitutions per site and the 1,836,182 concatenated bases for penalized

247 likelihood estimation, with cross validation to optimize the smoothing parameter which

248 quantifies the deviation from the molecular clock.

249 We used linear regression to determine the relationship between the estimated divergence

250 time for each node and the gene and site concordance factors in the inferred trees from each

251 locus type in the SISRS analysis, the gene trees and the species tree based on single-copy

252 orthologs, and the locus trees and partitioned maximum likelihood tree based on single-copy

253 orthologs. We also tested for a correlation between the concordance factors and the estimated

254 rates of molecular evolution at each branch. We repeated these regressions using estimated

255 divergence times from Thomson et al. (2020).

\section{Results}

258 A very large number of informative sites for turtle phylogenomics

259 We analyzed publicly available genome assemblies to extract single copy orthologs from 19

260 turtles representing 13 out of 14 extant families and 7 of 8 superfamilies, plus 3 outgroup taxa

261 (Table 1). On average, 4,609 (83\%, range 1,911-5,099) conserved tetrapod orthologs were

262 complete in the genome assemblies, $112(2.1 \%$, range 7-496) were duplicated, $170(3.2 \%$, range

263 55-485) were fragmented, and 419 (7.9\%, range 143-2,907) were missing. Fifty-five percent of

264 conserved tetrapod orthologs were missing from the genome assembly of the trionychid Apalone

265 spinifera; therefore, we omitted this species from downstream analyses of single-copy orthologs

266 due to concerns about assembly quality, of which BUSCO is known to be a reliable indicator

267 (Waterhouse et al. 2018). Each single-copy ortholog was present in an average of 19 out of 21 


\section{GABLE AND BYARS ET AL.}

species, averaged 1,902 bp in length, and contained an average of 53\% variable sites and 26\%

269 parsimony-informative sites. Alignment length was correlated with the number of parsimony

270 informative sites $\left(\mathrm{R}^{2}=0.69, \mathrm{P}=2.2 \mathrm{e}-16\right.$, Fig. 3a). GC content was consistent across the single-

271 copy orthologs and averaged $48 \%$ (standard deviation $6.2 \%$, Fig. 3b), as was average pairwise

272 Kimura 2-parameter distance (mean 0.154, median 01.39, standard deviation 0.072, Fig. 3c). The

273 subset of 2,513 alignments $\geq 1,500$ bp in length had very similar characteristics to the complete

274 set of loci; the average large locus length was 1,903 bp and the relationship between alignment

275 length and the number of parsimony-informative sites held $\left(\mathrm{R}^{2}=0.73, \mathrm{P}=2.2 \mathrm{e}-16\right)$.

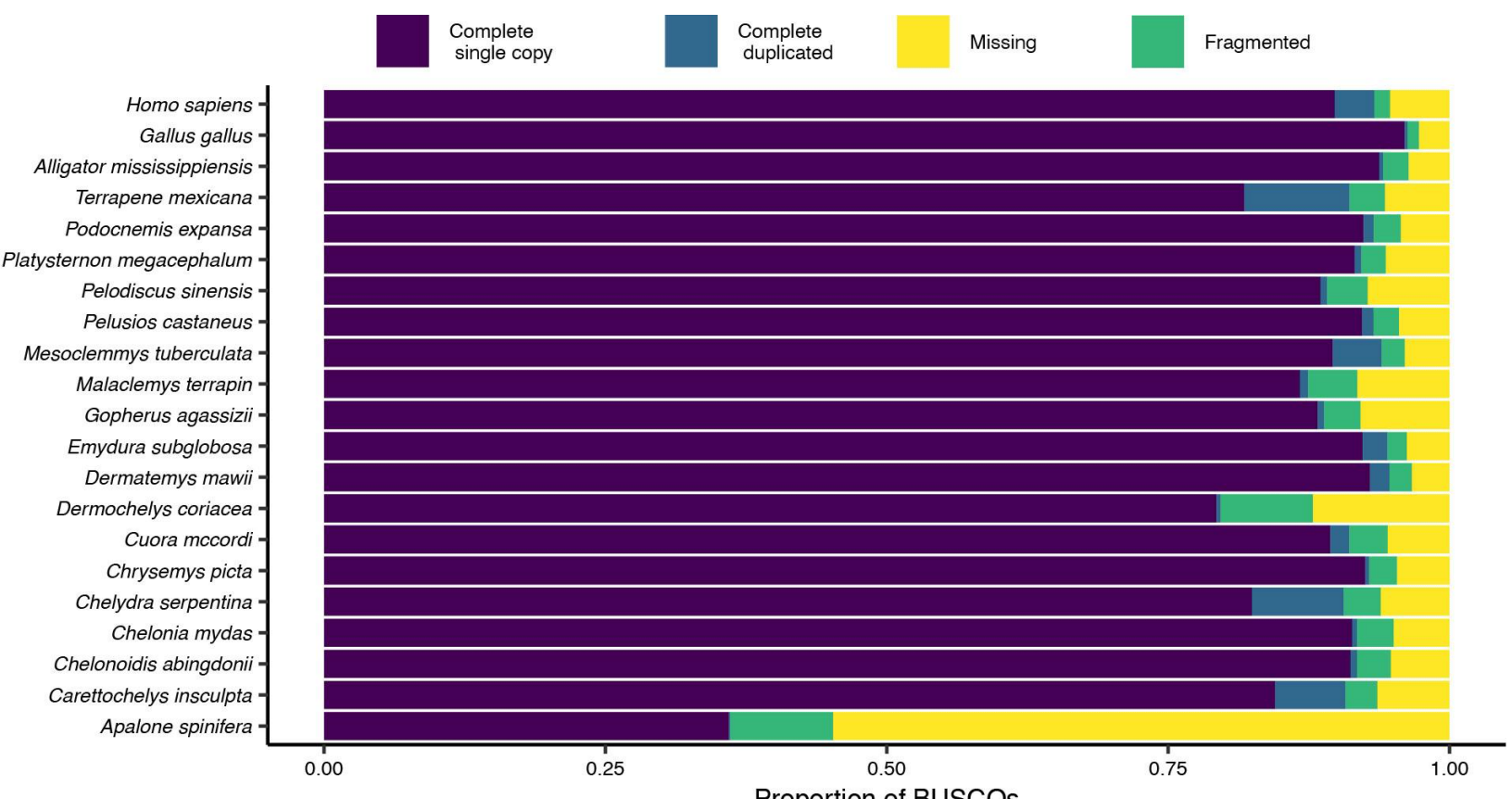

278 Figure 2. Summary of single-copy orthologs. Percent of tetrapod orthologs from orthoDBv9 present in each

279 genome assembly as complete single copy, complete duplicated, fragmented, or missing. 


\section{PHYLOGENOMIC HETEROGENEITY IN TURTLES}

a

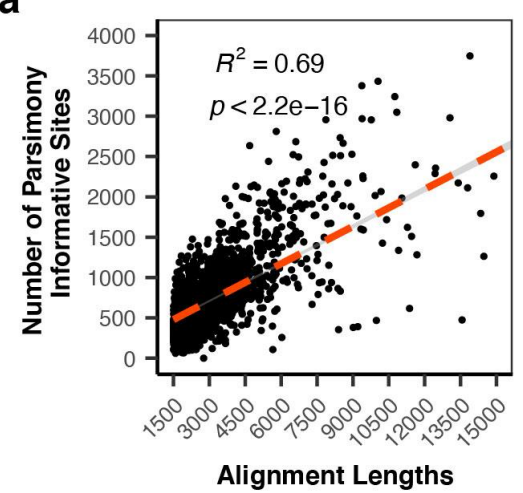

b

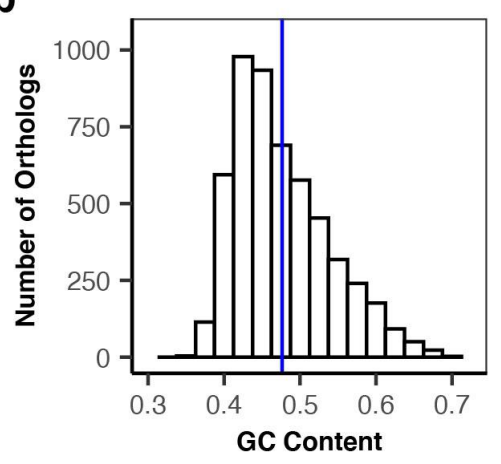

C

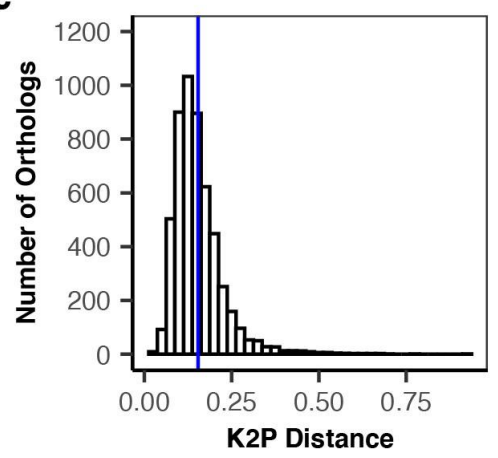

Figure 3. Description of single copy ortholog alignments. (a) Scatterplot showing the relationship between alignment length and the number of parsimony informative sites for trimmed alignments $\geq 1,500 \mathrm{bp}$. (b) Histogram showing the distribution of GC content in all single copy orthologs after trimming. Solid vertical line represents the mean value (0.476). (c) Histogram showing the distribution of average pairwise Kimura 2-parameter (K2P) distances for single copy orthologs after trimming. Solid vertical line represents the mean value (0.154). and 14 families (Supplementary Data). Based on FastQC results, we selected 32 of the datasets for further analysis ranging from $\sim 1-70 \mathrm{X}$ post-trimming coverage. The composite genome consisted of 5,555,666 contigs with an N50 of $2.4 \mathrm{Mb}$. We successfully mapped 302,355,876 bases to $2,110,354$ contigs in the composite genome which covered $80 \%$ of the painted turtle genome (Table 2), and called 1,655,675 parsimony-informative biallelic SNPs when allowing missing data for up to two species at a given site. The number of parsimony-informative biallelic SNPs was much larger in mapped datasets containing only cryptodires (2,787,072 bases) and testudinoideans (4,696,637 bases) (Supplementary Data), as expected based on Literman et al.

297 (2021). We collected the highest number of biallelic SNPs from intergenic regions (746,724 bases), followed by introns (533,336 bases) and CDS (136,823 bases). The relatively low 


\section{GABLE AND BYARS ET AL.}

300 (137) is likely due to a small number of annotations for these features in the western painted

301 turtle genome.

Table 2. Summary of data mapped to the western painted turtle (Chrysemys picta) genome annotations.

\begin{tabular}{|c|c|c|c|}
\hline Locus Type & Annotated & Based & Percent of \\
Genome & SISRS & Mapped \\
\hline All Mapped & $2,365,766,571$ & $302,355,876$ & $12.78 \%$ \\
\hline Intergenic & $1,382,145,112$ & $161,445,195$ & $11.68 \%$ \\
\hline Intronic & $812,010,672$ & $115,297,781$ & $14.20 \%$ \\
\hline lncRNA & $64,450,450$ & $9,451,244$ & $14.66 \%$ \\
\hline Genic Other & $65,764,053$ & $9,052,113$ & $13.76 \%$ \\
\hline CDS & $33,486,790$ & $5,718,074$ & $17.08 \%$ \\
\hline 3'-UTR & $8,034,514$ & $1,510,783$ & $18.80 \%$ \\
\hline 5'-UTR & $5,096,255$ & 708,214 & $13.90 \%$ \\
\hline Pseudogenic & 670,023 & 59,058 & $8.81 \%$ \\
\hline smRNA & 133,894 & 20,076 & $14.99 \%$ \\
\hline & & & \\
\hline & & & \\
\hline & & & \\
\hline & & & \\
\hline & & & \\
\hline & & & \\
\hline & & & \\
\hline
\end{tabular}

Bioinformatic analysis of turtle genomes yielded well-resolved species relationships

The inferred relationships among turtle lineages based on single-copy orthologs were consistent with previous studies using phylogenomic markers (Crawford et al. 2015; Shaffer et al. 2017), with full statistical support for all resulting branches based on posterior probabilities in the multilocus coalescent tree, and bootstrap replicates and SH-like approximate likelihood in the 


\section{PHYLOGENOMIC HETEROGENEITY IN TURTLES}

310 supermatrix tree (Fig. 4, Supplementary Fig. 1, Supplementary Fig. 2). Hereafter we refer to

311 these results collectively as the turtle species tree. The turtle species tree supports the reciprocal

312 monophyly of Pleurodira and Cryptodira. Within cryptodires, there is a split between the

313 Trionychoidea (soft-shelled turtles) and the Durocryptodira (Crawford et al. 2015), and a further

314 subdivision of Durocryptodira into the Testudinoidea (pond turtles, big-headed turtles,

315 geoemyds, and tortoises) and Americhelydia (sea turtles, snapping turtles, mud turtles; Joyce et

316 al. 2013). Within the Testudinoidea, the turtle species tree supports Testuguria (geoemyds and

317 tortoises; Joyce et al. 2004), and a Platysternon + Emydidae clade ("Platyemidae"; Crawford et

318 al. 2015). 


\section{GABLE AND BYARS ET AL.}

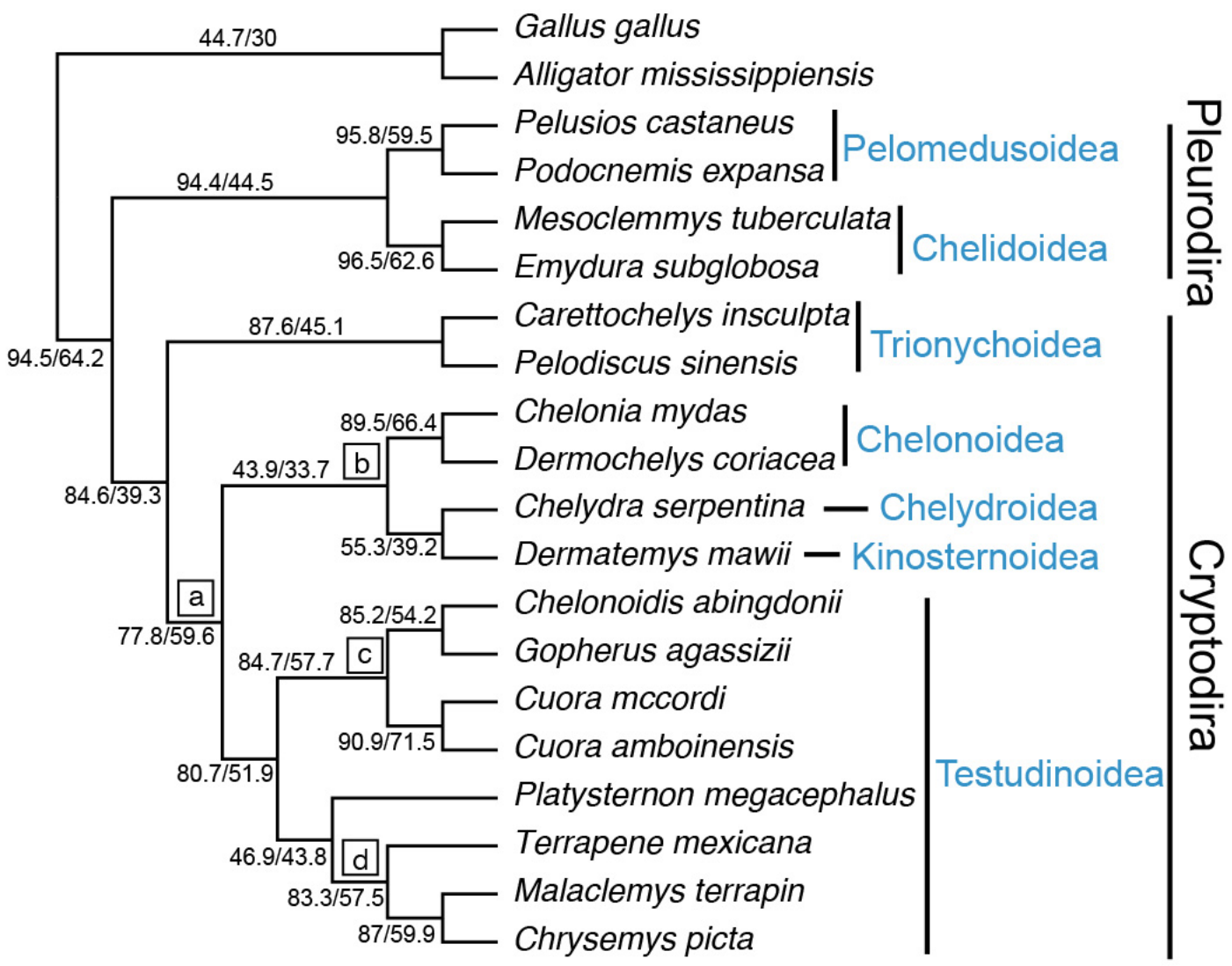

322 Figure 4. Multilocus coalescent species tree of turtles based on high-confidence single-copy orthologs.

323 Topology of the species tree inferred from 2,513 single-copy orthologs $\geq 1,500$ bp in length. All branches received

$324100 \%$ local posterior support. Numbers on branches indicate gene and site concordance factors before and after the

325 slash, respectively. Superfamilies are indicated by vertical text. Key clades are labelled with letters in boxes. (a)

326 Durocryptodira, (b) Americhelydia, (c) Testuguria, (d) Emydidae. Additional outgroup Homo sapiens not shown.

Maximum likelihood and quartets trees from the mapped data, particularly for all-

329 mapped, CDS, introns, and intergenic regions, were consistent with the species tree based on

330 single-copy orthologs (Supplementary Fig. 3-12) although this was highly dependent on the

331 amount of allowed missing data and gapless sites, and was variable between the taxonomic

332 specificity of the data used to map sites (Supplementary Data). When there was disagreement 


\section{PHYLOGENOMIC HETEROGENEITY IN TURTLES}

333 between trees from mapped regions and the species tree, the difference was often in the

334 placement of Platysternon (Fig. 5). For instance, when allowing zero missing gapless parsimony-

335 informative sites from the all-turtle data, 5' UTR placed Platysternon as the outgroup of

336 Geoemydidae + Testudinidae. When allowing up to two missing gapless parsimony-informative

337 sites from the all-turtle data, pseudogenic regions (426 sites) also placed Platysternon as the

338 outgroup of Geoemydidae + Testudinidae, while smRNA (137 sites) agreed with the species tree

339 and all-mapped topologies. Meanwhile, at multiple levels of allowed missing data the

340 Testudinoidea-only mapped sites weakly supported Platysternon as the sister taxon to

341 Geoemydidae + Testudinidae + Emydidae (Supplementary Data).
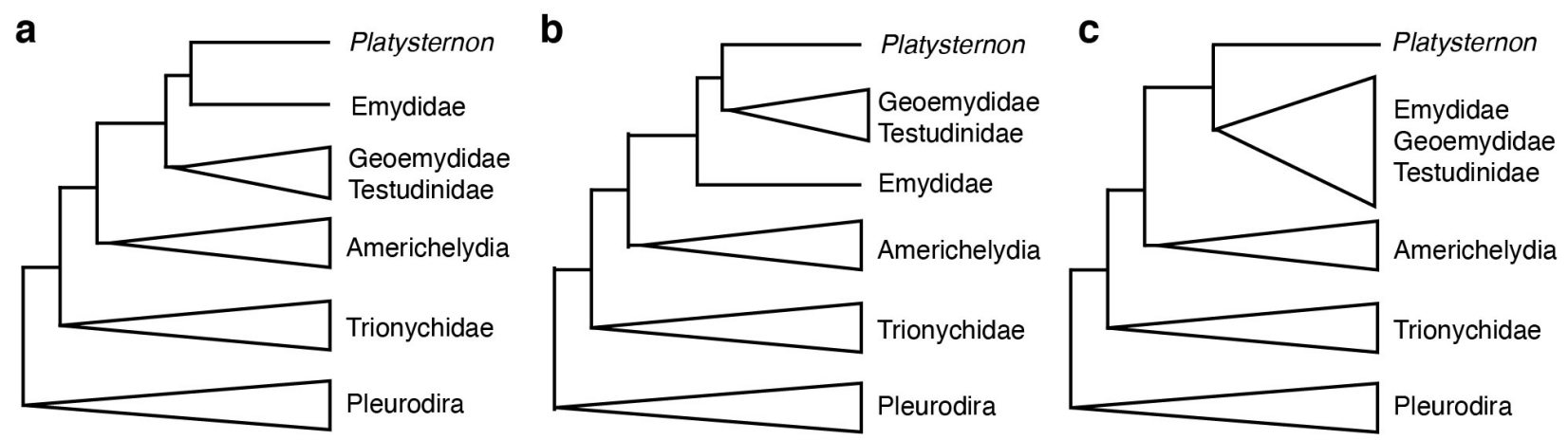

Figure 5. Alternative topologies in turtle phylogenetics based on markers from different regions of the

345 genome. (a) Platysternon as the sister taxon to Emydidae to the exclusion of Testuguria (Geoemydidae +

346 Testudinidae), consistent with the turtle species tree (current study), Crawford et al. (2015), and Shaffer et al.

347 (2017). (b) Platysternon as the sister taxon to Testuguria with the exclusion of Emydidae, consistent with $17 \%$ of the

348 single-copy ortholog genes trees, mapped 5'-UTR parsimony-informative sites data with zero missing data, mapped

349 pseudogene parsimony-informative sites when allowing 2 missing taxa per site, and Krenz et al. (2005). (c)

350 Platysternon as the sister taxon to Geoemydidae + Testudinidae + Emydidae, consistent with $20 \%$ of single-copy

351 ortholog gene trees and Shaffer et al. (1997). 


\section{GABLE AND BYARS ET AL.}

Heterogeneity is prevalent at well resolved nodes in the turtle phylogeny

354 While results from our multilocus and biallelic sites analyses of turtles are consistent and largely

355 in agreement with other studies based on 100\% branch support, some genomic regions, either

356 from single-copy orthologs or from genome feature annotations, yielded genealogies conflicting

357 with the turtle species tree. While most gene trees from single-copy orthologs matched the

358 species tree based on normalized RF distances, there was a long tail to this distribution indicating

359 gene trees with greater dissimilarity (Fig. 6a). Quartet trees using mapped data from CDS,

360 introns, and intergenic regions were consistent with both the single-copy ortholog species tree

361 and a quartet tree derived from the all-mapped data, except for pseudogenic DNA, 5' UTR, and

362 smRNA which deviated from the all-mapped data in terms of RF distances (Supplementary Fig.

$36313)$.

We also found a high degree of heterogeneity based on gene and site concordance factors

365 throughout the turtle phylogeny (Fig. 1, Supplementary Fig. 1, Supplementary Fig. 2). For

366 instance, while local posterior probabilities were uniformly $100 \%$ across branches of the

367 multilocus coalescent species tree, gene concordance factors ranged from 44 to 97 and site

368 concordance factors ranged from 30 to 71 (Fig. 6b-d). Gene and site concordance factors based

369 on the multilocus species tree were correlated with each other (Figure $6 \mathrm{~b} ; \mathrm{R}^{2}=0.55, \mathrm{P}=0.0002$ )

370 and with branch lengths (Fig. $6 \mathrm{c}-\mathrm{d} ; \mathrm{R}^{2}=0.88,3.88 \mathrm{e}-09$ and $\mathrm{R}^{2}=0.49, \mathrm{P}=0.0007$, respectively). We

371 also estimated gene and site concordance factors based on the locus trees with respect to the

372 maximum likelihood tree inferred from the concatenated supermatrix, and found a similar range

373 of gene concordance factors (34 to 92) and site concordance factors (30 to 71 ) despite $100 \%$

374 bootstrap support, as well as a strong correlation between the two concordance measures 


\section{PHYLOGENOMIC HETEROGENEITY IN TURTLES}

$375\left(\mathrm{R}^{2}=0.60, \mathrm{P}=0.0001\right)$. Gene and site concordance factors based on the concatenated partitioned

376 maximum likelihood tree were also correlated with branch lengths $\left(\mathrm{R}^{2}=0.90,1.02 \mathrm{e}-09\right.$ and

$377 \mathrm{R}^{2}=0.58, \mathrm{P}=0.0001$, respectively).

378
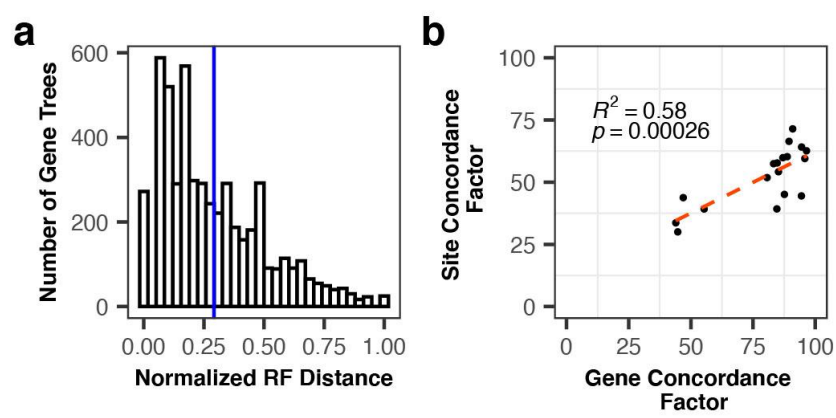

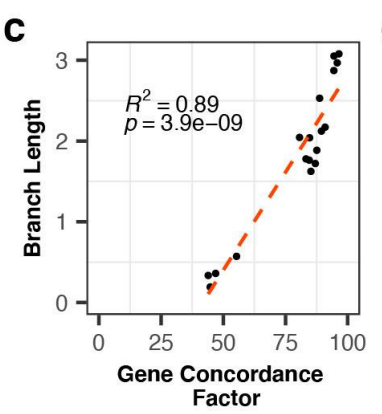

379

380

381

382

383

384

Figure 6. Phylogenomic heterogeneity in turtles. (a) Histogram of RF distances between gene trees and species tree. (b-c) Concordance factors calculated using single-copy ortholog alignments, their gene trees, and the multilocus coalescent species tree. Results based on locus trees and concatenated supermatrix maximum likelihood tree are in Supplementary Materials. (b) Scatterplot depicting the relationship between gene and site concordance factors at each node. (c) Scatterplot depicting the relationship between gene concordance factor and branch length. (d) Scatterplot depicting the relationship between site concordance factor and branch length.

The root of the turtle species tree was assigned a relatively large gene concordance factor (94.5) and site concordance factor (64.2), suggesting strong support for reciprocally monophyletic Pleurodira and Cryptodira across genes and sites. We rejected incomplete lineage sorting at the root of the turtle phylogeny and the roots of Cryptodira $(\mathrm{P}=8.05 \mathrm{E}-04)$ and Pleurodira ( $\mathrm{P}=2.32 \mathrm{E}-04)$. The placement of Trionychoidea as sister taxon to all other cryptodires was assigned relatively large gene concordance factor (84.56) but small site concordance factor (39.26), suggesting that there are a large number of sites supporting alternative bifurcations. One area with particularly high discordance (i.e. relatively small gene concordance factor $=46.9$ and site concordance factor=43.8) was the placement of the big-headed turtle (Platysternon) with 


\section{GABLE AND BYARS ET AL.}

respect to Emydidae, for which we could not reject incomplete lineage sorting (see Table 3, Fig.

Table 3. Gene concordance factors for nodes in the species tree phylogeny where incomplete lineage sorting was not rejected.

\begin{tabular}{|l|l|l|l|l|l|}
\hline Clade description & Node & $\begin{array}{l}\text { Number of possible } \\
\text { supporting gene } \\
\text { trees }\end{array}$ & $\begin{array}{l}\text { Percent of gene } \\
\text { Gene concordance } \\
\text { factor }\end{array}$ & $\begin{array}{l}\text { Percent of gene } \\
\text { trees supporting } \\
\text { trees supporting } \\
\text { second alternative } \\
\text { topology }\end{array}$ \\
\hline topology
\end{tabular}

401 Results from the replicate BEAST analyses consistently underestimated turtle divergence times

402 with respect to results from the penalized likelihood analysis as well as those of Thomson et al.

403 (2020), even though the TMRCA for all taxa (i.e. the time of the mammal-reptile split) was

404 consistent (Supplementary Data). Across locus types from the SISRS analysis, we found that site

405 concordance factors were not strongly correlated with estimated node ages, regardless of whether

406 or not we used our estimated divergence times or those from Thomson et al. (2021) (Fig. 7,

407 Supplementary Fig. 14). For most locus types the relationship between site concordance factor

408 and divergence time at a given node trended slightly negatively, suggesting a pattern of higher

409 concordance at younger nodes, although these comparisons were non-significant (Fig. 7b, 


\section{PHYLOGENOMIC HETEROGENEITY IN TURTLES}

$410 \mathrm{P}>0.1)$. The one exception to this trend was for pseudogenes, for which the relationship between

411 site concordance factors and estimated divergence times trended positive albeit without

412 significance or with only moderate significance $(\mathrm{P}=0.072$ using Thomson et al. 2020's

413 divergence time estimates). The relationship between site concordance factors and estimated

414 DNA substitution rates trended slightly positive for all locus types from the SISRS analysis, but

415 was non-significant in all comparisons (Supplementary Fig. 15).

416 We also found a negative trend in the relationship between site concordance factor and

417 divergence time at a given node based on both the multilocus species tree and the supermatrix

418 tree based on single-copy orthologs, similar to the patterns observed using the locus types from

419 SISRS (Fig. 8a-c; Supplementary Fig. 16). However, we found a positive (albeit non-significant)

420 trend in the relationship between the estimated divergence time at a given node and its gene

421 concordance factor when using both trees derived from single-copy orthologs (Fig. 8e-g;

422 Supplementary Fig. 16). There was no clear relationship between the site concordance factor of a

423 given node and the estimated substitution rate at the preceding branch (Fig. 8d), and a

424 moderately significant positive trend $(\mathrm{P} \leq 0.05)$ for the relationship between the gene concordance

425 factor of a given node and the estimated substitution rate at the preceding branch (Fig. 8h;

426 Supplementary Fig. 16). 


\section{GABLE AND BYARS ET AL.}
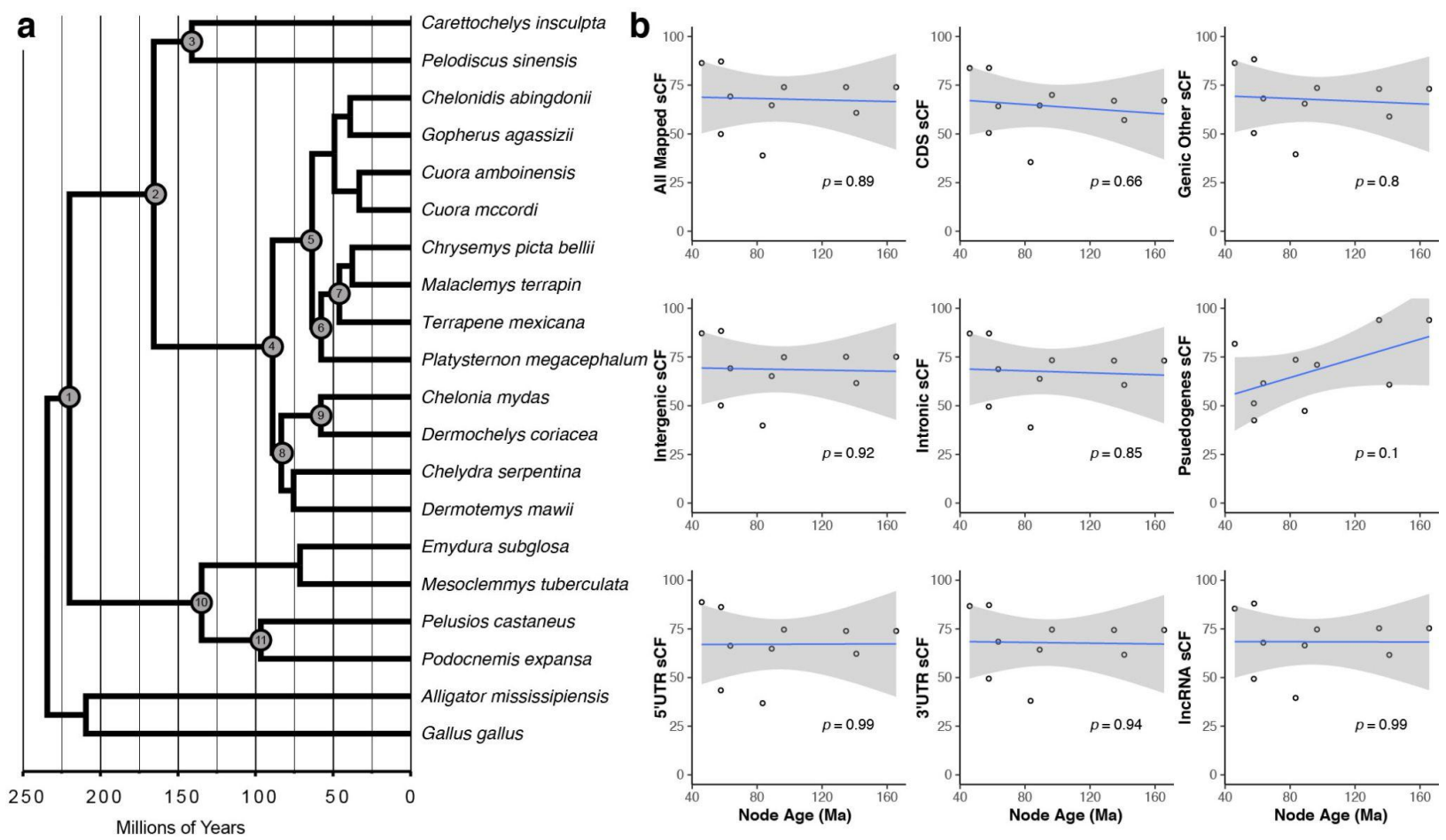

428 Figure 7. Site concordance and estimated node ages in turtle evolution. (a) Chronogram showing estimated

429 divergence times in millions of years based on penalized likelihood and fossil calibrations of labelled nodes. 1.

430 Testudines, 2. Cryptodira, 3. Trionychoidea, 4. Durocryptodira, 5. Geoemydidae, 6. Platyemidae, 7. Emydidae, 8.

431 Americhelydia, 9. Chelonioidea, 10. Pleurodira, 11. Pelomedusoidea. (b) Site concordance factors (sCF) for each

432 node in phylogenies inferred from nine locus types and the estimated node age in millions of years ago (Ma) based

433 on penalized likelihood. 


\section{PHYLOGENOMIC HETEROGENEITY IN TURTLES}
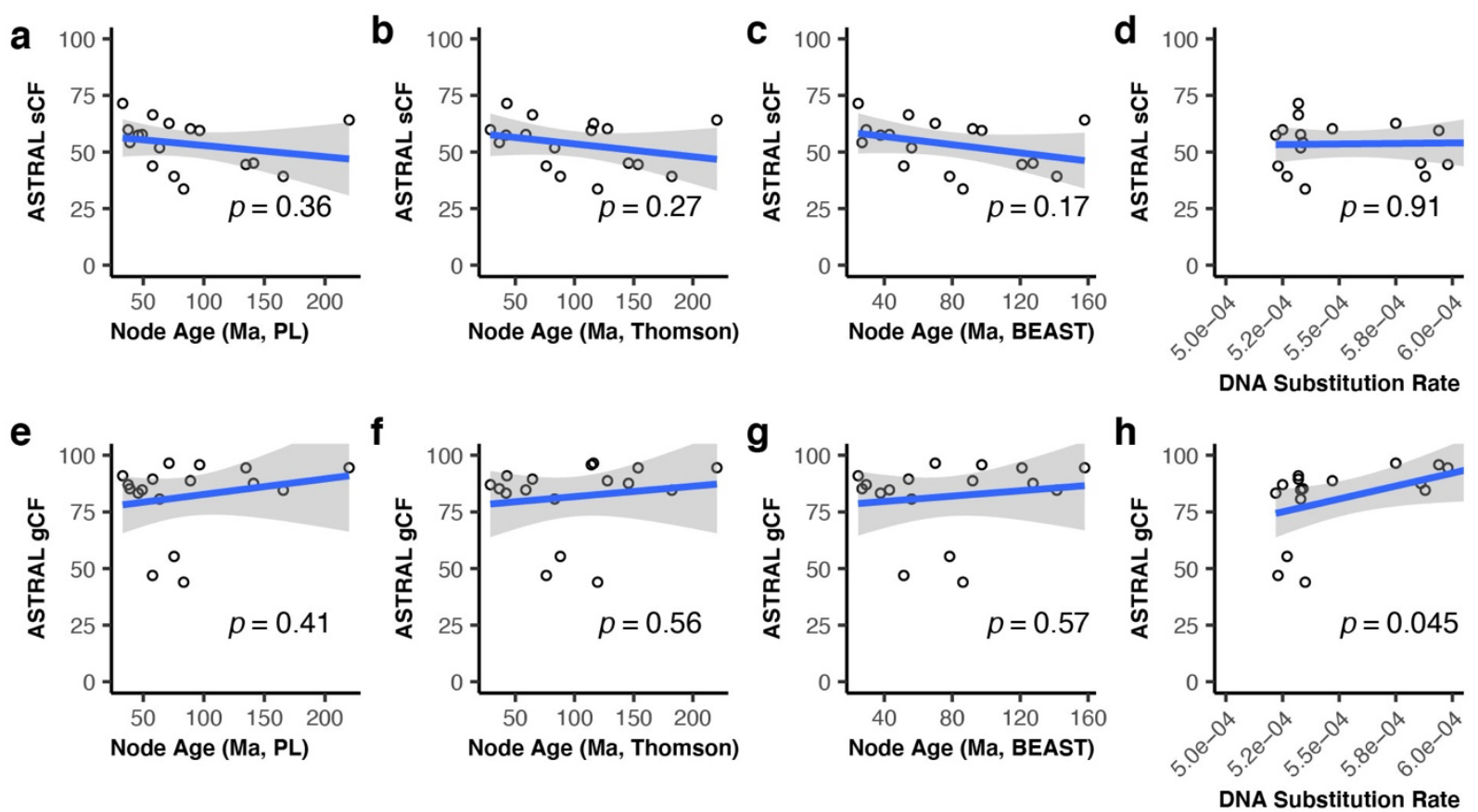

Figure 8. Concordance at genes and sites, estimated node ages, and DNA substitution rates in turtle evolution.

437 Results shown are from comparisons of single-copy ortholog alignments and their gene trees to the multilocus

438 coalescent species tree inferred using ASTRAL. There was a negative trend between site concordance factors (sCF)

439 and estimated node age (in millions of years or Ma) based on penalized likelihood (PL) from the current study (a),

440 estimated node ages in Ma from Thomson et al. (2020) (b), estimated node ages in Ma from the BEAST analysis in

441 the current study (c), and no discernable trend between $\mathrm{SCF}$ and estimated DNA substitution rates in terms of

442 substitutions per site per Ma (d). There was a positive trend between gene concordance factors (gCF) and estimated

443 node ages (e, f, g) and a moderately significant positive relationship between gene concordance factors and

444 estimated DNA substitution rates (h). Results from comparisons of single-copy ortholog alignments, locus trees, and

445 the maximum likelihood tree inferred from the concatenated supermatrix are shown in Supplementary Materials.

\section{Discussion}

448 While heterogeneity has been recognized as an important feature of phylogenomic datasets, and

449 the exploration of the patterns and sources of gene tree and site discordance has become an

450 essential step when reconstructing the branching order of life with molecular data (Kumar et al. 


\section{GABLE AND BYARS ET AL.}

451 2012; Minh et al. 2020a), to our knowledge there has not been an assessment of phylogenomic

452 heterogeneity in turtles until now. Here, we have constructed a robust dataset based on whole

453 genomes with the goal of disentangling conflicting hypotheses about turtle relationships. Single-

454 copy orthologs yielded more than double the variation and triple the number of parsimony

455 informative sites for turtles compared to other high-throughput methods (Crawford et al. 2015;

456 Shaffer et al. 2017). In addition, the mapped data included millions of parsimony informative

457 sites that were further parsed by locus type. We identified splits in the turtle phylogeny and

458 regions of the turtle genome associated with gene tree and site heterogeneity, and investigated

459 potential sources of this discordance. We found that heterogeneity in turtle phylogenomics can

460 be explained by a combination of factors, such as incomplete lineage sorting and lack of gene

461 informativeness, and has contributed to conflicting results in phylogenetic studies of turtles. The

462 results of our study demonstrate that heterogeneity can be rampant, even in phylogenomic

463 datasets which have yielded fully resolved species trees.

Gene tree-species tree discordance in turtles may be partly due to an overall slow

465 substitution rate compared to other amniotes (Tollis et al. 2018; Gemmell et al. 2020), resulting

466 in a lack of gene informativeness at more recent divergences. We found no significant

467 relationship between site concordance and node age across most locus types, a uniform

468 distribution of site concordances across the range of estimated DNA substitution rates across

469 most annotated genomic regions, and that the average pairwise Kimura 2-parameter distances

470 across single-copy orthologs were also fairly uniform. This suggests relatively consistent

471 substitution rates across the turtle genome, as well as across turtle evolution. Where we did

472 estimate elevated DNA substitution rates along branches in the turtle phylogeny, we found that

473 gene concordance factors were significantly larger. This suggests that shifts in the rate of 


\section{PHYLOGENOMIC HETEROGENEITY IN TURTLES}

474 evolution may lead to a comparatively rapid accumulation of mutations during certain time

475 periods, resulting in more gene trees in agreement with the species phylogeny. Meanwhile, the

476 gene concordances at older nodes were larger (albeit non-significantly), suggesting that

477 heterogeneity at more recent nodes may be more driven by stochastic error due to limited

478 information at slowly evolving loci. Our results are consistent with the longstanding prediction

479 that slowly evolving loci, which includes much of the turtle genome, provide disproportionally

480 greater phylogenetic information for older splits (Townsend et al. 2008).

481 Interestingly, site concordance in pseudogenes was positively associated (albeit non-

482 significantly) with node age, suggesting that even slightly elevated substitution rates in some

483 noncoding regions of the turtle genome can lead to more informative sites supporting the species

484 tree topology at older nodes. This is somewhat different from what was reported in Literman et

485 al. (2021), who showed that noncoding regions such as introns and intergenic regions supported

486 more recent splits in the mammalian phylogeny, and may be again related to the fact that

487 estimated substitution rates in mammals are consistently higher than for other amniotes such as

488 turtle and crocodilians. However, our inferences from pseudogenes are based on a relatively

489 small number of parsimony informative sites (427). More comprehensive annotations for

490 noncoding regions of turtle genomes are needed to investigate this further.

491 Our tests for incomplete lineage sorting reveal that population-level processes likely also

492 have played a key role at multiple stages of turtle diversification. In particular, the phylogenetic

493 position of Platysternon has been highly contentious (Joyce et al. 2004, 2021), with early

494 molecular studies in disagreement (Shaffer et al. 1997; Krenz et al. 2005; Parham et al. 2006;

495 Barley et al. 2010). Our turtle species tree results mirror Crawford et al. (2015) and Shaffer et al.

496 (2017), fully supporting Platysternon as the sister taxon to Emydidae. However, only 47\% of 


\section{GABLE AND BYARS ET AL.}

497 single-copy ortholog gene trees are in agreement with a Platysternon + Emydidae clade, and

$49837 \%$ of gene trees support only two alternative relationships: of these, $17 \%$ of gene trees support

499 Platysternon as the sister taxon to Testuguria (Geoemydidae + Testudinidae) following Krenz et

500 al. (2005), and 20\% support Platysternon as the sister taxon to Testudinoidea (Emydidae +

501 Geoemydidae + Testudinidae) following Shaffer et al. (1997). The proportion of gene trees

502 supporting these alternative topologies was similar according to our chi-square test (Table 3),

503 and supports incomplete lineage sorting rather than introgression or statistical noise (Green et al.

504 2010). As these loci support published alternative hypotheses concerning the relationship of

505 Platysternon to other Testudinoidea, we suggest that incomplete lineage sorting is a previously

506 unrecognized driver of conflicting phylogenetic results in the higher classification of turtles.

\section{Acknowledgements}

509 This research was funded by the National Science Foundation (\#1812291 to RL) and by startup

510 funds provided to MT by the School of Informatics, Computing, and Cyber Systems at Northern

511 Arizona University. We would like to acknowledge the Monsoon computing cluster at Northern

512 Arizona University (https://nau.edu/high-performance-computing/) for providing the

513 computational resources necessary to carry out this study.

\section{Data Availability}

516 The bioinformatic workflows used to extract and analyze single-copy orthologs from genome

517 assemblies are available at https://github.com/mibyars/busco_phylo_pipeline. Data generated for

518 this study including alignments and trees are available at the Harvard Dataverse

519 (https://doi.org/10.7910/DVN/WYQRPC). 


\section{PHYLOGENOMIC HETEROGENEITY IN TURTLES}

\section{Literature Cited}

522 Barley A.J., Spinks P.Q., Thomson R.C., Shaffer H.B. 2010. Fourteen nuclear genes provide phylogenetic resolution for difficult nodes in the turtle tree of life. Mol. Phylogenet. Evol. 55:1189-1194.

525

Boisvert S., Laviolette F., Corbeil J. 2010. Ray: Simultaneous Assembly of Reads from a Mix of High-Throughput Sequencing Technologies. J. Comput. Biol. 17:1519-1533.

Borowiec M.L. 2016. AMAS: a fast tool for alignment manipulation and computing of summary statistics. PeerJ. 4:e1660.

Bouckaert R., Vaughan T.G., Barido-Sottani J., Duchêne S., Fourment M., Gavryushkina A., Heled J., Jones G., Kühnert D., Maio N.D., Matschiner M., Mendes F.K., Müller N.F., Ogilvie H.A., Plessis L. du, Popinga A., Rambaut A., Rasmussen D., Siveroni I., Suchard M.A., Wu C.-H., Xie D., Zhang C., Stadler T., Drummond A.J. 2019. BEAST 2.5: An advanced software platform for Bayesian evolutionary analysis. PLOS Comput. Biol. 15:e1006650.

Burbrink F.T., Grazziotin F.G., Pyron R.A., Cundall D., Donnellan S., Irish F., Keogh J.S., Kraus F., Murphy R.W., Noonan B., Raxworthy C.J., Ruane S., Lemmon A.R., Lemmon E.M., Zaher H. 2020. Interrogating Genomic-Scale Data for Squamata (Lizards, Snakes, and Amphisbaenians) Shows no Support for Key Traditional Morphological Relationships. Syst. Biol. 69:502-520.

Chifman J., Kubatko L. 2014. Quartet Inference from SNP Data Under the Coalescent Model. Bioinformatics. 30:3317-3324.

Cooper E.D. 2014. Overly simplistic substitution models obscure green plant phylogeny. Trends 
GABLE AND BYARS ET AL.

Plant Sci. 19:576-582.

Cox C.J., Li B., Foster P.G., Embley T.M., Civáň P. 2014. Conflicting Phylogenies for Early Land Plants are Caused by Composition Biases among Synonymous Substitutions. Syst.

Crawford N.G., Faircloth B.C., McCormack J.E., Brumfield R.T., Winker K., Glenn T.C. 2012.

Crawford N.G., Parham J.F., Sellas A.B., Faircloth B.C., Glenn T.C., Papenfuss T.J., Henderson Phylogenet. Evol. 83:250-257.

Donoghue P.C.J., Benton M.J. 2007. Rocks and clocks: calibrating the Tree of Life using fossils and molecules. Trends Ecol. Evol. 22:424-431.

Dudchenko O., Batra S.S., Omer A.D., Nyquist S.K., Hoeger M., Durand N.C., Shamim M.S.,

Edwards S.V., Xi Z., Janke A., Faircloth B.C., McCormack J.E., Glenn T.C., Zhong B., Wu S.,

562 Faircloth B.C., Sorenson L., Santini F., Alfaro M.E. 2013. A Phylogenomic Perspective on the testing the multispecies coalescent model: A valuable paradigm for phylogenomics. Mol. 


\section{PHYLOGENOMIC HETEROGENEITY IN TURTLES}

566 Galtier N., Daubin V. 2008. Dealing with incongruence in phylogenomic analyses. Philos. Trans.

R. Soc. B Biol. Sci. 363:4023-4029.

Gemmell N.J., Rutherford K., Prost S., Tollis M., Winter D., Macey J.R., Adelson D.L., Suh A., F.J., Flicek P., Petersen B., Kang L., Michalak P., Buckley T.R., Wilson M., Cheng Y., Miller H., Schott R.K., Jordan M.D., Newcomb R.D., Arroyo J.I., Valenzuela N., Hore 2020. The tuatara genome reveals ancient features of amniote evolution. Nature. 584:403-409.

Green R.E., Krause J., Briggs A.W., Maricic T., Stenzel U., Kircher M., Patterson N., Li H., Zhai W., Fritz M.H.-Y., Hansen N.F., Durand E.Y., Malaspinas A.-S., Jensen J.D., MarquesBonet T., Alkan C., Prüfer K., Meyer M., Burbano H.A., Good J.M., Schultz R., AximuPetri A., Butthof A., Höber B., Höffner B., Siegemund M., Weihmann A., Nusbaum C., Lander E.S., Russ C., Novod N., Affourtit J., Egholm M., Verna C., Rudan P., Brajkovic D., Kucan Ž., Gušic I., Doronichev V.B., Golovanova L.V., Lalueza-Fox C., Rasilla M. de la, Fortea J., Rosas A., Schmitz R.W., Johnson P.L.F., Eichler E.E., Falush D., Birney E., Mullikin J.C., Slatkin M., Nielsen R., Kelso J., Lachmann M., Reich D., Pääbo S. 2010. A Draft Sequence of the Neandertal Genome. Science. 328:710-722.

Hime P.M., Lemmon A.R., Lemmon E.C.M., Prendini E., Brown J.M., Thomson R.C., Kratovil 
GABLE AND BYARS ET AL.

J.D., Noonan B.P., Pyron R.A., Peloso P.L.V., Kortyna M.L., Keogh J.S., Donnellan

S.C., Mueller R.L., Raxworthy C.J., Kunte K., Ron S.R., Das S., Gaitonde N., Green

D.M., Labisko J., Che J., Weisrock D.W. 2021. Phylogenomics Reveals Ancient Gene

Tree Discordance in the Amphibian Tree of Life. Syst. Biol. 70:48-66.

Irisarri I., Baurain D., Brinkmann H., Delsuc F., Sire J.-Y., Kupfer A., Petersen J., Jarek M.,

Meyer A., Vences M., Philippe H. 2017. Phylotranscriptomic consolidation of the jawed vertebrate timetree. Nat. Ecol. Evol. 1:1370-1378.

Jarvis E.D., Mirarab S., Aberer A.J., Li B., Houde P., Li C., Ho S.Y.W., Faircloth B.C., Nabholz 


\section{PHYLOGENOMIC HETEROGENEITY IN TURTLES}

$$
\text { Science. 346:1320-1331. }
$$

613 Joyce W.G. 2007. Phylogenetic Relationships of Mesozoic Turtles. Bull. Peabody Mus. Nat. Hist. 48:3-102.

Joyce W.G., Anquetin J., Cadena E.-A., Claude J., Danilov I.G., Evers S.W., Ferreira G.S., Gentry A.D., Georgalis G.L., Lyson T.R., Pérez-García A., Rabi M., Sterli J., Vitek N.S.,

Joyce W.G., Parham J.F., Gauthier J.A. 2004. Developing a protocol for the conversion of rankbased taxon names to phylogenetically defined clade names, as exemplified by turtles. J.

Joyce W.G., Parham J.F., Lyson T.R., Warnock R.C.M., Donoghue P.C.J. 2013. A divergence dating analysis of turtles using fossil calibrations: an example of best practices. J.

Kalyaanamoorthy S., Minh B.Q., Wong T.K.F., von Haeseler A., Jermiin L.S. 2017.

Katoh K., Standley D.M. 2013. MAFFT Multiple Sequence Alignment Software Version 7: $14: 587-589$. Improvements in Performance and Usability. Mol. Biol. Evol. 30:772-780.

632 Kubatko L.S., Degnan J.H. 2007. Inconsistency of Phylogenetic Estimates from Concatenated Data under Coalescence. Syst. Biol. 56:17-24. 
GABLE AND BYARS ET AL.

Truth in Phylogenomics. Mol. Biol. Evol. 29:457-472.

Kumar S., Stecher G., Li M., Knyaz C., Tamura K. 2018. MEGA X: Molecular Evolutionary

Genetics Analysis across Computing Platforms. Mol. Biol. Evol. 35:1547-1549.

Lanfear R., Calcott B., Ho S.Y.W., Guindon S. 2012. Partitionfinder: combined selection of partitioning schemes and substitution models for phylogenetic analyses. Mol. Biol. Evol.

Langmead B., Salzberg S.L. 2012. Fast gapped-read alignment with Bowtie 2. Nat. Methods.

\section{3}

644

645

$$
9: 357-359 \text {. }
$$

Lemmon A.R., Emme S.A., Lemmon E.M. 2012. Anchored Hybrid Enrichment for Massively High-Throughput Phylogenomics. Syst. Biol. 61:727-744.

Literman R., Schwartz R. 2021. Genome-Scale Profiling Reveals Noncoding Loci Carry Higher Proportions of Concordant Data. Mol. Biol. Evol.

Lopes F., Oliveira L.R., Kessler A., Beux Y., Crespo E., Cárdenas-Alayza S., Majluf P., Sepúlveda M., Brownell R.L. Jr., Franco-Trecu V., Páez-Rosas D., Chaves J., Loch C., Robertson B.C., Acevedo-Whitehouse K., Elorriaga-Verplancken F.R., Kirkman S.P., Peart C.R., Wolf J.B.W., Bonatto S.L. 2021. Phylogenomic Discordance in the Eared Seals is best explained by Incomplete Lineage Sorting following Explosive Radiation in the Southern Hemisphere. Syst. Biol. 70:786-802.

Lynch M. 2007. The Origins of Genome Architecture. Sinauer Associates Sunderland, MA.

Minh B.Q., Hahn M.W., Lanfear R. 2020a. New Methods to Calculate Concordance Factors for Phylogenomic Datasets. Mol. Biol. Evol. 37:2727-2733.

Minh B.Q., Schmidt H.A., Chernomor O., Schrempf D., Woodhams M.D., von Haeseler A., Lanfear R. 2020b. IQ-TREE 2: New Models and Efficient Methods for Phylogenetic 


\section{PHYLOGENOMIC HETEROGENEITY IN TURTLES}

Inference in the Genomic Era. Mol. Biol. Evol. 37:1530-1534.

Mölder F., Jablonski K.P., Letcher B., Hall M.B., Tomkins-Tinch C.H., Sochat V., Forster J., Lee S., Twardziok S.O., Kanitz A., Wilm A., Holtgrewe M., Rahmann S., Nahnsen S., Köster J. 2021. Sustainable data analysis with Snakemake.

Morales-Briones D.F., Kadereit G., Tefarikis D.T., Moore M.J., Smith S.A., Brockington S.F., https://f1000research.com/articles/10-33. Timoneda A., Yim W.C., Cushman J.C., Yang Y. 2021. Disentangling Sources of Gene Tree Discordance in Phylogenomic Data Sets: Testing Ancient Hybridizations in Amaranthaceae s.1. Syst. Biol. 70:219-235.

Pamilo P., Nei M. 1988. Relationships between gene trees and species trees. Mol. Biol. Evol. 5:568-583.

Parham J.F., Feldman C.R., Boore J.L. 2006. The complete mitochondrial genome of the enigmatic bigheaded turtle (Platysternon): description of unusual genomic features and the reconciliation of phylogenetic hypotheses based on mitochondrial and nuclear DNA. BMC Evol. Biol. 6:11.

Prum R.O., Berv J.S., Dornburg A., Field D.J., Townsend J.P., Lemmon E.M., Lemmon A.R. 2015. A comprehensive phylogeny of birds (Aves) using targeted next-generation DNA sequencing. Nature. 526:569-573.

Quesada V., Freitas-Rodríguez S., Miller J., Pérez-Silva J.G., Jiang Z.-F., Tapia W., SantiagoFernández O., Campos-Iglesias D., Kuderna L.F.K., Quinzin M., Álvarez M.G., Carrero D., Beheregaray L.B., Gibbs J.P., Chiari Y., Glaberman S., Ciofi C., Araujo-Voces M., Mayoral P., Arango J.R., Tamargo-Gómez I., Roiz-Valle D., Pascual-Torner M., Evans B.R., Edwards D.L., Garrick R.C., Russello M.A., Poulakakis N., Gaughran S.J., Rueda 
GABLE AND BYARS ET AL.

681

682

683

684

685

686

687

688

689

690

691

692

\section{3}

694

695

696

697

698

699

700

701

702

703

D.O., Bretones G., Marquès-Bonet T., White K.P., Caccone A., López-Otín C. 2019.

Giant tortoise genomes provide insights into longevity and age-related disease. Nat. Ecol. Evol. 3:87-95.

Quinlan A.R., Hall I.M. 2010. BEDTools: a flexible suite of utilities for comparing genomic features. Bioinformatics. 26:841-842.

Rambaut A., Drummond A.J., Xie D., Baele G., Suchard M.A. 2018. Posterior summarization in Bayesian phylogenetics using Tracer 1.7. Syst. Biol. 67:901-904.

Robinson D.F., Foulds L.R. 1981. Comparison of phylogenetic trees. Math. Biosci. 53:131-147.

Rokas A., Williams B.L., King N., Carroll S.B. 2003. Genome-scale approaches to resolving incongruence in molecular phylogenies. Nature. 425:798-804.

Sanderson M.J. 1997. A nonparametric approach to estimating divergence times in the absence of rate constancy. Mol. Biol. Evol.

Sanderson M.J. 2002. Estimating absolute rates of molecular evolution and divergence times: a penalized likelihood approach. Mol. Biol. Evol. 19:101-109.

Sanderson M.J. 2003. r8s: inferring absolute rates of molecular evolution and divergence times in the absence of a molecular clock. Bioinformatics. 19:301-302.

Sayyari E., Mirarab S. 2016. Fast Coalescent-Based Computation of Local Branch Support from Quartet Frequencies. Mol. Biol. Evol. 33:1654-1668.

Schwartz R.S., Harkins K.M., Stone A.C., Cartwright R.A. 2015. A composite genome approach to identify phylogenetically informative data from next-generation sequencing. BMC Bioinformatics. 16:193.

Shaffer H.B., McCartney-Melstad E., Near T.J., Mount G.G., Spinks P.Q. 2017. Phylogenomic analyses of 539 highly informative loci dates a fully resolved time tree for the major 


\section{PHYLOGENOMIC HETEROGENEITY IN TURTLES}

clades of living turtles (Testudines). Mol. Phylogenet. Evol. 115:7-15.

705

706

707

708

709

710

711

712

713

714

715

716

717

718

719

720

721

722

723

724

725

726

Shaffer H.B., Meylan P., McKnight M.L. 1997. Tests of turtle phylogeny: molecular, morphological, and paleontological approaches. Syst. Biol. 46:235-268.

Shaffer H.B., Minx P., Warren D.E., Shedlock A.M., Thomson R.C., Valenzuela N., Abramyan J., Amemiya C.T., Badenhorst D., Biggar K.K., Borchert G.M., Botka C.W., Bowden R.M., Braun E.L., Bronikowski A.M., Bruneau B.G., Buck L.T., Capel B., Castoe T.A., Czerwinski M., Delehaunty K.D., Edwards S.V., Fronick C.C., Fujita M.K., Fulton L., Graves T.A., Green R.E., Haerty W., Hariharan R., Hernandez O., Hillier L.W., Holloway A.K., Janes D., Janzen F.J., Kandoth C., Kong L., de Koning A.P.J., Li Y., Literman R., McGaugh S.E., Mork L., O’Laughlin M., Paitz R.T., Pollock D.D., Ponting C.P., Radhakrishnan S., Raney B.J., Richman J.M., St John J., Schwartz T., Sethuraman A., Spinks P.Q., Storey K.B., Thane N., Vinař T., Zimmerman L.M., Warren W.C., Mardis E.R., Wilson R.K. 2013. The western painted turtle genome, a model for the evolution of extreme physiological adaptations in a slowly evolving lineage. Genome Biol. 14:R28.

Shimodaira H. 2002. An Approximately Unbiased Test of Phylogenetic Tree Selection. Syst. Biol. 51:492-508.

Singhal S., Colston T.J., Grundler M.R., Smith S.A., Costa G.C., Colli G.R., Moritz C., Pyron R.A., Rabosky D.L. 2021. Congruence and Conflict in the Higher-Level Phylogenetics of Squamate Reptiles: An Expanded Phylogenomic Perspective. Syst. Biol. 70:542-557.

Stamatakis A. 2014. RAxML version 8: a tool for phylogenetic analysis and post-analysis of large phylogenies. Bioinformatics. 30:1312-1313.

Steenwyk J.L., Buida T.J. III, Labella A.L., Li Y., Shen X.-X., Rokas A. 2021. PhyKIT: a 
GABLE AND BYARS ET AL.

broadly applicable UNIX shell toolkit for processing and analyzing phylogenomic data.

Swofford D.L. 2003. PAUP*. Phylogenetic Analysis Using Parsimony (*and Other Methods).

Thomson R.C., Spinks P.Q., Shaffer H.B. 2021. A global phylogeny of turtles reveals a burst of climate-associated diversification on continental margins. Proc. Natl. Acad. Sci. 118.

Tollis M., Denardo D.F., Cornelius J.A., Dolby G.A., Edwards T., Henen B.T., Karl A.E.,

Tollis M., Hutchins E.D., Stapley J., Rupp S.M., Eckalbar W.L., Maayan I., Lasku E., Infante C.R., Dennis S.R., Robertson J.A., May C.M., Crusoe M.R., Bermingham E., Denardo

Townsend J.P., López-Giráldez F, Friedman R. 2008. The phylogenetic informativeness of nucleotide and amino acid sequences for reconstructing the vertebrate tree. J. Mol. Evol.

Wang Z., Pascual-Anaya J., Zadissa A., Li W., Niimura Y., Wang J., Huang Z., Li C., White S., Xiong Z., Fang D., Wang B., Ming Y., Chen Y., Zheng Y., Kuraku S., Pignatelli M., 


\section{PHYLOGENOMIC HETEROGENEITY IN TURTLES}

2013. The draft genomes of soft-shell turtle and green sea turtle yield insights into the development and evolution of the turtle-specific body plan. Nat. Genet. 45:701-706.

752 Waterhouse R.M., Seppey M., Simão F.A., Manni M., Ioannidis P., Klioutchnikov G.,

753 Kriventseva E.V., Zdobnov E.M. 2018. BUSCO Applications from Quality Assessments to Gene Prediction and Phylogenomics. Mol. Biol. Evol. 35:543-548.

755 Wolf Y.I., Rogozin I.B., Grishin N.V., Koonin E.V. 2002. Genome trees and the tree of life.

756 Trends Genet. TIG. 18:472-479.

757 Zdobnov E.M., Tegenfeldt F., Kuznetsov D., Waterhouse R.M., Simão F.A., Ioannidis P., Seppey M., Loetscher A., Kriventseva E.V. 2017. OrthoDB v9.1: cataloging evolutionary and functional annotations for animal, fungal, plant, archaeal, bacterial and viral

$760 \quad$ orthologs. Nucleic Acids Res. 45:D744-D749.

761 Zhang C., Rabiee M., Sayyari E., Mirarab S. 2018. ASTRAL-III: polynomial time species tree reconstruction from partially resolved gene trees. BMC Bioinformatics. 19:153. 